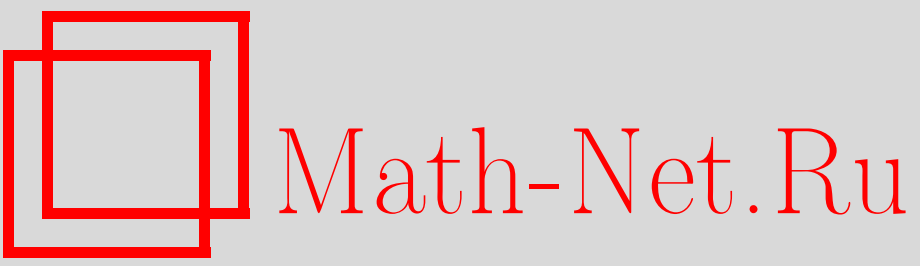

О. И. Мохов, Пары Лакса для согласованных нелокальных гамильтоновых операторов гидродинамического типа, УМН, 2002, том 57, выпуск 6, 189-190

DOI: https://doi.org/10.4213/rm586

Использование Общероссийского математического портала Math-Net.Ru подразумевает, что вы прочитали и согласны с пользовательским соглашением

http://www . mathnet.ru/rus/agreement

Параметры загрузки:

IP: 35.173 .219 .149

26 апреля 2023 г., 18:19:37 


\title{
ПАРЫ ЛАКСА ДЛЯ СОГЛАСОВАННЫХ НЕЛОКАЛЬНЫХ ГАМИЛЬТОНОВЫХ ОПЕРАТОРОВ ГИДРОДИНАМИЧЕСКОГО ТИПА
}

\author{
О.И. Мохов
}

Получены нелинейные уравнения для неособых пар согласованных нелокалшных гамильтоновых операторов гидродинамического типа и доказана интегрируемость этих уравнений методом обратной задачи рассеяния. Для этих уравнений найдена пара Лакса со спектральным параметром. В [1] Ферапонтов ввел и изучил общие нелокальные гамильтоновы операторы гидродинамического типа, а именно, гамильтоновы операторы вида

$$
P^{i j}=g^{i j}(u(x)) \frac{d}{d x}+b_{k}^{i j}(u(x)) u_{x}^{k}+\sum_{\alpha=1}^{L} \varepsilon_{\alpha}\left(w^{\alpha}\right)_{k}^{i}(u(x)) u_{x}^{k}\left(\frac{d}{d x}\right)^{-1} \circ\left(w^{\alpha}\right)_{s}^{j}(u(x)) u_{x}^{s},
$$

где $\operatorname{det}\left(g^{i j}(u)\right) \not \equiv 0, \varepsilon_{\alpha}= \pm 1$. Пара метрик $g_{1}^{i j}(u)$ и $g_{2}^{i j}(u)$ назьвается неособой, если собственные значения этой пары метрик, т.е. корни уравнения $\operatorname{det}\left(g_{1}^{i j}(u)-\lambda g_{2}^{i j}(u)\right)=0$, различны. В [2] доказано, что произвольная неособая пара метрик согласована тогда и только тогда, когда существуют локальные координаты $u=\left(u^{1}, \ldots, u^{N}\right)$ такие, что обе метрики диагональны в этих координатах и имеют следующий специальньй вид: $g_{2}^{i j}(u)=g^{i}(u) \delta^{i j}$ и $g_{1}^{i j}(u)=f^{i}\left(u^{i}\right) g^{i}(u) \delta^{i j}$, где $f^{i}\left(u^{i}\right), i=1, \ldots, N,-$ функции одной переменной (вообще говоря, комплексные). Если гамильтоновы операторы вида (1) согласованы, то и их метрики согласованы [2]. Более того, в [2] было доказано также, что если 1) пара метрик $g_{1}^{i j}(u)$ и $g_{2}^{i j}(u)$ является неособой, 2) метрики $g_{1}^{i j}(u), g_{2}^{i j}(u)$ и афффиноры $\left(w_{1}^{\beta}\right)_{j}^{i}(u),\left(w_{2}^{\alpha}\right)_{j}^{i}(u)$ одновременно диагонализируются в некоторой области локальных координат, то соответствующие гамильтоновы операторы согласованы тогда и только тогда, когда согласованы их метрики. Здесь мы доказьваем в некотором смысле обратную теорему.

ТЕОРема 1. Если пара метрик $g_{1}^{i j}(u)$ и $g_{2}^{i j}(u)$ является неособой, то гамильтоновь операторы вида (1) согласованы тогда и только тогда, когда 1) их метрики $g_{1}^{i j}(u)$ и $g_{2}^{i j}(u)$ согласованы, 2) метрики $g_{1}^{i j}(u), g_{2}^{i j}(u)$ и аффиноры $\left(w_{1}^{\beta}\right)_{j}^{i}(u),\left(w_{2}^{\alpha}\right)_{j}^{i}(u)$ одновременно диагонализируются в некоторой области локальных координат.

Рассмотрим произвольную неособую пару согласованных нелокальных гамильтоновых операторов $P_{1}^{i j}$ и $P_{2}^{i j}$ вида $(1)$, т.е. мы предполагаем, что пара метрик $g_{1}^{i j}(u)$ и $g_{2}^{i j}(u)$ является неособой.

Теорема 2. Неособые пары согласованных нелокальных гамильтоновых операторов вида (1) описьваются следующими совместными интегрируемыми нелинейными системами:

$$
\begin{gathered}
\epsilon_{2}^{i} \frac{\partial \beta_{i j}}{\partial u^{i}}+\epsilon_{2}^{j} \frac{\partial \beta_{j i}}{\partial u^{j}}+\sum_{s \neq i, s \neq j} \epsilon_{2}^{s} \beta_{s i} \beta_{s j}+\sum_{\alpha=1}^{L_{2}} \varepsilon_{2, \alpha} H_{2, i}^{\alpha} H_{2, j}^{\alpha}=0, \quad i \neq j, \\
\frac{\partial \beta_{i j}}{\partial u^{k}}=\beta_{i k} \beta_{k j}, \quad i \neq j, \quad i \neq k, \quad j \neq k, \quad \frac{\partial H_{2, j}^{\alpha}}{\partial u^{i}}=\beta_{i j} H_{2, i}^{\alpha}, \\
\epsilon_{2}^{i} f^{i}\left(u^{i}\right) \frac{\partial \beta_{i j}}{\partial u^{i}}+\frac{1}{2} \epsilon_{2}^{i}\left(f^{i}\right)^{\prime} \beta_{i j}+\epsilon_{2}^{j} f^{j}\left(u^{j}\right) \frac{\partial \beta_{j i}}{\partial u^{j}}+\frac{1}{2} \epsilon_{2}^{j}\left(f^{j}\right)^{\prime} \beta_{j i} \\
+\sum_{s \neq i, s \neq j} \epsilon_{2}^{s} f^{s}\left(u^{s}\right) \beta_{s i} \beta_{s j}+\sum_{\beta=1}^{L_{1}} \varepsilon_{1, \beta} H_{1, i}^{\beta} H_{1, j}^{\beta}=0, \quad \frac{\partial H_{1, j}^{\beta}}{\partial u^{i}}=\beta_{i j} H_{1, i}^{\beta}, \quad i \neq j,
\end{gathered}
$$

әде $f^{i}\left(u^{i}\right), i=1, \ldots, N,-$ произвольнье заданнье функции одной переменной.

Работа вьполнена при финансовой поддержке Фонда Александра фон Гумболдта (Германия), а также РФФИ (грант № 02-01-00803) и фонда INTAS (грант № 99-1782). 
В соответствии с теоремой 1 существуют локальные координаты такие, что в этих координа$\operatorname{Tax}$

$$
g_{2}^{i j}(u)=g^{i}(u) \delta^{i j}, g_{1}^{i j}(u)=f^{i}\left(u^{i}\right) g^{i}(u) \delta^{i j},\left(w_{2}^{\alpha}\right)_{j}^{i}(u)=\left(w_{2}^{\alpha}\right)^{i}(u) \delta_{j}^{i},\left(w_{1}^{\beta}\right)_{j}^{i}(u)=\left(w_{1}^{\beta}\right)^{i}(u) \delta_{j}^{i} .
$$

Более того, в соответствии с той же самой теоремой 1 любая пара гамилштоновых операторов такого вида является согласованной. Таким образом, достаточно рассмотреть условия, что операторы этого специального вида являются гамильтоновьми операторами. Введем классические обозначения

$$
g^{i}(u)=\frac{\epsilon_{2}^{i}}{\left(H_{i}(u)\right)^{2}}, \quad d s^{2}=\sum_{i=1}^{N} \epsilon_{2}^{i}\left(H_{i}(u)\right)^{2}\left(d u^{i}\right)^{2}, \quad \beta_{i k}(u)=\frac{1}{H_{i}(u)} \frac{\partial H_{k}}{\partial u^{i}}, i \neq k,
$$

где $H_{i}(u)$ - коэффициенты Ламе, а $\beta_{i k}(u)$ - коэффициенты вращения, $\epsilon_{2}^{i}= \pm 1$. В рассматриваемом “диагональном" случае условие, что оператор $P_{2}^{i j}$ является гамильтоновым, эквивалентно уравнениям Гаусса-Кодацци для подмногообразий с плоской нормальной связностью и голономной сетью линий кривизны (см. [3], [4]). Введем функции $H_{1, i}^{\beta}(u), H_{2, i}^{\alpha}(u), 1 \leqslant \beta \leqslant L_{1}$, $1 \leqslant \alpha \leqslant L_{2}$, такие, что $\left(w_{2}^{\alpha}\right)^{i}(u)=H_{2, i}^{\alpha}(u) / H_{i}(u),\left(w_{1}^{\beta}\right)^{i}(u)=H_{1, i}^{\beta}(u) / H_{i}(u)$. Пару Лакса со спектральным параметром для системы (2)-(4) можно получить из линейной задачи для системы $(2),(3)$, описьвающей все подмногообразия с плоской нормальной связностью и голономной сетью линий кривизны и эквивалентной условиям совместности для следующей линейной системы:

$\frac{\partial \varphi_{i}}{\partial u^{i}}=-\sum_{k \neq i} \frac{\sqrt{\epsilon_{2}^{k}}}{\sqrt{\epsilon_{2}^{i}}} \beta_{k i} \varphi_{k}+\sum_{\alpha=1}^{L_{2}} \frac{\sqrt{\varepsilon_{2, \alpha}}}{\sqrt{\epsilon_{2}^{i}}} H_{2, i}^{\alpha} \psi^{\alpha}, \frac{\partial \varphi_{i}}{\partial u^{j}}=\frac{\sqrt{\epsilon_{2}^{i}}}{\sqrt{\epsilon_{2}^{j}}} \beta_{i j} \varphi_{j}, \frac{\partial \psi^{\alpha}}{\partial u^{i}}=-\frac{\sqrt{\varepsilon_{2, \alpha}}}{\sqrt{\epsilon_{2}^{i}}} H_{2, i}^{\alpha} \varphi_{i}$,

где $i \neq j$. Условие, что оператор $P_{1}^{i j}+\lambda P_{2}^{i j}$ является гамильтоновым для любого $\lambda$, эквивалентно системе (2)-(4) для метрики $\left(\lambda+f^{i}\left(u^{i}\right)\right) g^{i}(u) \delta^{i j}$ и афффиноров $\left(w_{1}^{\beta}\right)_{j}^{i}(u), 1 \leqslant \beta \leqslant L_{1}$, и $\sqrt{\lambda}\left(w_{2}^{\alpha}\right)_{j}^{i}(u), 1 \leqslant \alpha \leqslant L_{2}$. В этом случае линейная задача (7) превращается в пару Лакса со спектральным параметром $\lambda$ для общей неособой пары произвольных согласованных нелокальных гамильтоновых операторов гидродинамического типа:

$$
\begin{aligned}
& \frac{\partial \varphi_{i}}{\partial u^{i}}=-\sum_{k \neq i} \frac{\sqrt{\epsilon_{2}^{k}\left(\lambda+f^{k}\right)}}{\sqrt{\epsilon_{2}^{i}\left(\lambda+f^{i}\right)}} \beta_{k i} \varphi_{k}+\sum_{\alpha=1}^{L_{2}} \frac{\sqrt{\varepsilon_{2, \alpha} \lambda}}{\sqrt{\epsilon_{2}^{i}\left(\lambda+f^{i}\right)}} H_{2, i}^{\alpha} \psi^{\alpha}+\sum_{\beta=1}^{L_{1}} \frac{\sqrt{\varepsilon_{1, \beta}}}{\sqrt{\epsilon_{2}^{i}\left(\lambda+f^{i}\right)}} H_{1, i}^{\beta} \chi^{\beta}, \\
& \frac{\partial \varphi_{i}}{\partial u^{j}}=\frac{\sqrt{\epsilon_{2}^{i}\left(\lambda+f^{i}\right)}}{\sqrt{\epsilon_{2}^{j}\left(\lambda+f^{j}\right)}} \beta_{i j} \varphi_{j}, \quad \frac{\partial \psi^{\alpha}}{\partial u^{i}}=-\frac{\sqrt{\varepsilon_{2, \alpha} \lambda}}{\sqrt{\epsilon_{2}^{i}\left(\lambda+f^{i}\right)}} H_{2, i}^{\alpha} \varphi_{i}, \frac{\partial \chi^{\beta}}{\partial u^{i}}=-\frac{\sqrt{\varepsilon_{1, \beta}}}{\sqrt{\epsilon_{2}^{i}\left(\lambda+f^{i}\right)}} H_{1, i}^{\beta} \varphi_{i},
\end{aligned}
$$

где $i \neq j$. Наличие пары Лакса (8), (9) со спектральньм оператором доказьвает интегрируемость системы (2)-(4) для общей неособой пары согласованных нелокалшных гамильтоновых операторов гидродинамического типа.

\section{СПИСОК ЛИТЕРАТУРЫ}

[1] Е. В. Ферапонтов // Функц. анализ и его прил. 1991. Т. 25. № 3. С. 37-49. [2] О. И. Мохов. Функц. анализ и его прил. 2001. Т. 35. № 2. C. 24-36; math.DG/0005051 (2000). [3] E. V. Ferapontov // math.DG/9805012 (1998). [4] L. P. Eisenhart. Transformations of Surfaces. New York: Chelsea, 1962.

Центр нелинейных исследований при Институте теоретической физики им. Л. Д. Ландау РАН

Принято редколлегией E-mail: mokhov@mi.ras.ru, mokhov@landau.ac.ru 